\title{
On the Estimation of the Depth of Maximum of Extensive Air Showers Using the Steepness Parameter of the Lateral Distribution of Cherenkov Radiation
}

\author{
Ibrahim Rasekh, Davoud Purmohammad ${ }^{1}$ \\ Physics Department, Imam Khomeini International University, Qazvin, Iran
}

\begin{abstract}
Using Monte Carlo simulation of extensive air showers, we showed that the maximum depth of showers, $X_{\max }$ can be estimated using $P=Q(100) / Q(200)$, the ratio of Cherenkov photon densities at 100 and 200 meters from the shower core, which is known as the steepness parameter of the lateral distribution of Cherenkov radiation on the ground. A simple quadratic model has been fitted to a set of data from simulated extensive air showers, relating the steepness parameter and the shower maximum depth. Then the model has been tested on another set of simulated showers. The average difference between the actual maximum depth of the simulated showers and the maximum depth obtained from the lateral distribution of Cherenkov light is about $9 \mathrm{~g} / \mathrm{cm}^{2}$. In addition, possibility of a more direct estimation of the mass of the initial particle from $P$ has been investigated. An exponential relation between these two quantities has been fitted. Applying the model to another set of showers, we found that the average difference between the estimated and the actual mass of primary particles is less than 0.5 atomic mass unit.
\end{abstract}

Keywords: Cosmic Rays, Extensive Air Showers, Cherenkov Radiation

\section{Introduction}

Extremely energetic cosmic rays are accessible signs of phenomena such as supernova explosions, or active galactic nuclei in very distant parts of space. Determining characteristics such as energy and mass of cosmic rays helps us to identify their origin. Because of the very low flux of cosmic rays in very high energy, it is almost impossible to directly observe them outside the atmosphere. On the other hand, these energetic particles produce extensive showers of secondary charged particles in the atmosphere. The detection of these secondary charged particles, atmospheric fluorescence radiation, Cherenkov radiation, or

\footnotetext{
${ }^{1}$ Corresponding author
} 
radio waves emanating from the showers, are the main methods of detecting extensive air showers (EAS). Characteristics such as the size of a shower and the atmospheric depth at which the shower reaches its maximum size, which depend on the energy and type of the initial particle generating the shower, can be estimated by arrays of detectors on the ground. Studies on the structure of extensive air showers have shown that the maximum depth of showers correlate with their energy and primary mass $1,2,3,4,4,5,6$. Relatively direct measurement of the maximum depth of a shower is only possible with atmospheric fluorescence detector arrays which measure the amount of isotropic radiation from the passage of shower particles through the atmosphere, along the shower path [7]. On the other hand, particle detector arrays, or Cherenkov radiation detectors on the ground, can only observe the distribution of particles or Cherenkov radiation on a single observation level, i.e., the lateral distributions, and are not able to inspect the distribution of particles or radiation along the shower axis. Research has shown the correlation between the lateral distribution of Cherenkov radiation in an extensive air shower and the height of the shower maximum 8. The height can then be converted to the atmospheric depth by using an atmospheric density distribution model, e.g. section 6.1 of the reference [9]. Hence, the depth of the shower maximum can be estimated from the lateral distribution of its Cherenkov radiation. By analyzing the results form a large number of simulated extensive air showers, here we will establish an empirical relationship between the depth of the shower maximum and the steepness parameter of its Cherenkov radiation lateral distribution, with no need to the intermediate step of the height estimation. This provides a more accurate method for the estimation of the depth of shower maximum from the Cherenkov radiation lateral distribution. We also show that the mass of the primary particle can be estimated by using an empirical model that establishes an immediate relationship between the steepness parameter of the Cherenkov radiation distribution and the mass of the primary particle.

\section{Simulation}

9890 extensive air showers with energies from $10^{12}$ to $10^{17} \mathrm{eV}$, initiated by different primary particles, such as gamma ray, proton, iron, carbon, helium, silicon, oxygen, magnesium, aluminum, sulfur and scandium were produced by using CORSIKA simulation code [10. Most of these showers, which have been used for the establishment of the proposed methods, were generated by using QGSJETII model [11] for high energy, and GHEISHA model [12] for low energy hadronic interactions. In order to test the impact of the hadronic interaction models on the viability of our proposed methods, we used EPOS-LHC model [13] for high energy and UrQMD model [14] for low energy hadronic interactions to generate some other showers. The number of the simulated showers for different primary types and energyies for each part of the analysis is given in the relevant sections. No thinning has been applied. The simulations were set to generate longitudinal profiles of the particles in steps of $10 \mathrm{~g} / \mathrm{cm}^{2}$, and fit the profile to the Gaisser-Hillas type distribution specified in the CORSIKA documents [10]. The 
$t_{\text {max }}$ parameter of the Gaisser-Hillas distribution has been used as an estimation of the depth of maximum $\left(X_{\max }\right)$ of a shower. However, we have observed that, in some showers, the difference between evaluated $t_{\max }$ and the depth of the step corresponding to the maximum number of charged particles in the longitudinal output of CORSIKA is greater than the step size, which is $10 \mathrm{~g} / \mathrm{cm}^{2}$. In these cases, the sampled depth of maximum charged particles was taken as $X_{\max }$. The showers were simulated for the observation level of 675 meters above the sea level, which is the height of the Tunka-133 Cherenkov array [8]. This array is one of the few experiments in which the lateral distribution of Cherenkov radiation of showers is measured. The absorption of Cherenkov radiation in the atmosphere and the default quantum efficiency for detectors have been taken into account. No photon bunch size has been specified in the simulations inputs; hence, the default values were implemented $(\mathrm{CERSIZ}=0$.). By inspecting the data in the output files, we observed that the maximum number of Cherenkov photons in a bunch was 30 . The detector array responses were not simulated in this work. The showers produced in the simulations had zero zenith angle, and their energies were in the $10^{12} \mathrm{eV}$ to $10^{17} \mathrm{eV}$ interval. For shower energies less than $10^{16} \mathrm{eV}$, all Cherenkov photons within 201meter distance from shower cores have been simulated. An array of $81 \times 81$ flat detectors, with single detector area of $1 \mathrm{~m}^{2}$, and $5 \mathrm{~m}$ inter detector spacing has been implemented to reduce simulation time and output file size for higher energy showers. The outputs of a CORSIKA shower simulation contain information including the number and the location of the Cherenkov photons reaching the observation level, and the number of secondary particles in $10 \mathrm{~g} / \mathrm{cm}^{2}$ steps from the top to the bottom of the atmosphere. Therefore, the lateral distribution of Cherenkov radiation at the observation level and the depth of shower maximum have been calculated from the simulation results for each shower. To determine the lateral distribution of Cherenkov radiation of a shower, we divided the observation level into annuli of one meter in width around the shower core. The number of photons entered into each annulus was used to determine the number of photons per unit area, $Q(r)$ in each region of outer radius $r$ from the core. Then, the steepness of the lateral distribution $P=Q(100) / Q(200)$, in which $Q(100)$ and $Q(200)$ are photon densities at 100 and 200 meter form core respectively, has been calculated. An example of lateral distribution of Cherenkov radiation for $10^{14} \mathrm{eV}$ and $10^{15} \mathrm{eV}$ showers initiated by gamma ray, proton, helium, carbon and iron primaries is shown in Figure 1.

\section{Estimation of the maximum depth of extensive air showers using $P$}

Since the shower maximum depth is the main quantity used for the estimation of the primary particle masses, it would be interesting to remove the intermediate step of maximum height estimation and find an immediate relation between $X_{\max }$, and the steepness of the lateral distribution of Cherenkov radiation. Having tested several models, we chose a quadratic model to relate 


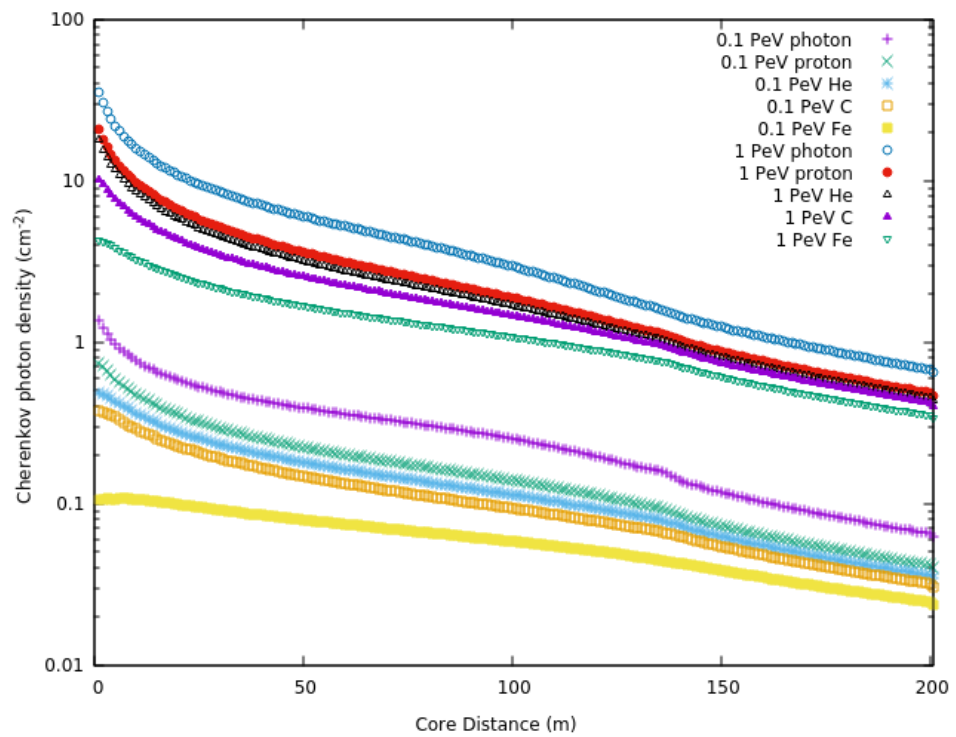

Figure 1: An example of lateral distribution of Cherenkov radiation for $10^{14} \mathrm{eV}$ and $10^{15}$ $\mathrm{eV}$ showers initiated by gamma ray (photon), proton, helium, carbon and iron primaries, simulated with QGSJETII+GHISHA models for an observation level 675 meter above sea level.

$X_{\max }$ and $P$, for its simplicity and suitable fit to the simulated data:

$$
X_{\max }=a P^{2}+b P+c
$$

The model is fitted to the data of 4340 simulated extensive air showers of different primarey particle types and energies. The number of the simulated showers for each type-energy used in the fit is given in Table 1. The fit and its parameters are presented in Figure 2. To test the model for $X_{\max }$ estimation in single showers, we tried it using another set of simulated showers as follows. The steepness parameter $P$ of each shower in the new set was obtained and the estimated depth of maximum, $X_{\max }^{\text {estimated }}$ was calculated by equation 1 . The actual depth of maximum, $X_{\max }^{\text {charged }}$ has also been obtained from the longitudinal charged particle distribution. The comparison of the two depths, for different primary masses and energies, has been represented in Figure 3. The number of simulated showers of different type-energies in Figure 3 is given in Table 2 . The average values of $P, X_{\text {max }}^{\text {estimated }}, X_{\max }^{\text {charged }}$, and their relative difference for the data represented in Figure 3 are given in Table 3 . The correlation between the estimated and the actual maxima of all showers in Figure 3 has been shown in Figure 4 . A histogram of the deviation of the estimated maxima is presented in Figure 4 . $b$. As Figure $4-b$ shows, the average and RMS of the differences are $9 \mathrm{~g} / \mathrm{cm}^{2}$, and $21 \mathrm{~g} / \mathrm{cm}^{2}$, respectively. 


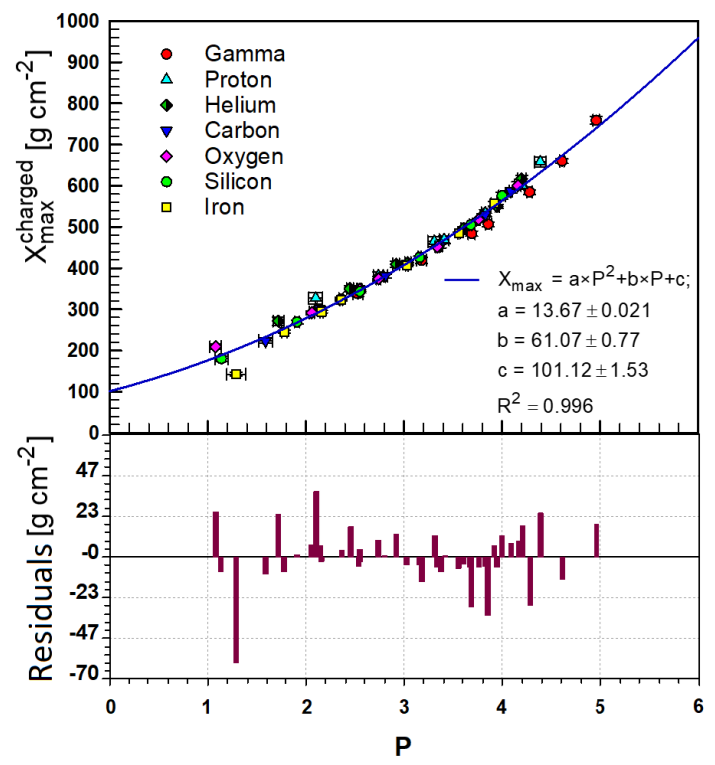

Figure 2: Above: Variation of shower maximum with the steepness of the Cherenkov radiation lateral distribution along with a quadratic model for 4340 simulated showers, generated from different primary particles type and energies. The data points represent average values for showers of the same type-energy. The number of showers for each type-energy is given in Table 1 The fit paremeters, and the coefficient of determination are also given. Below: The residual plot for the fit. The horizontal scales are the same for both plots. 

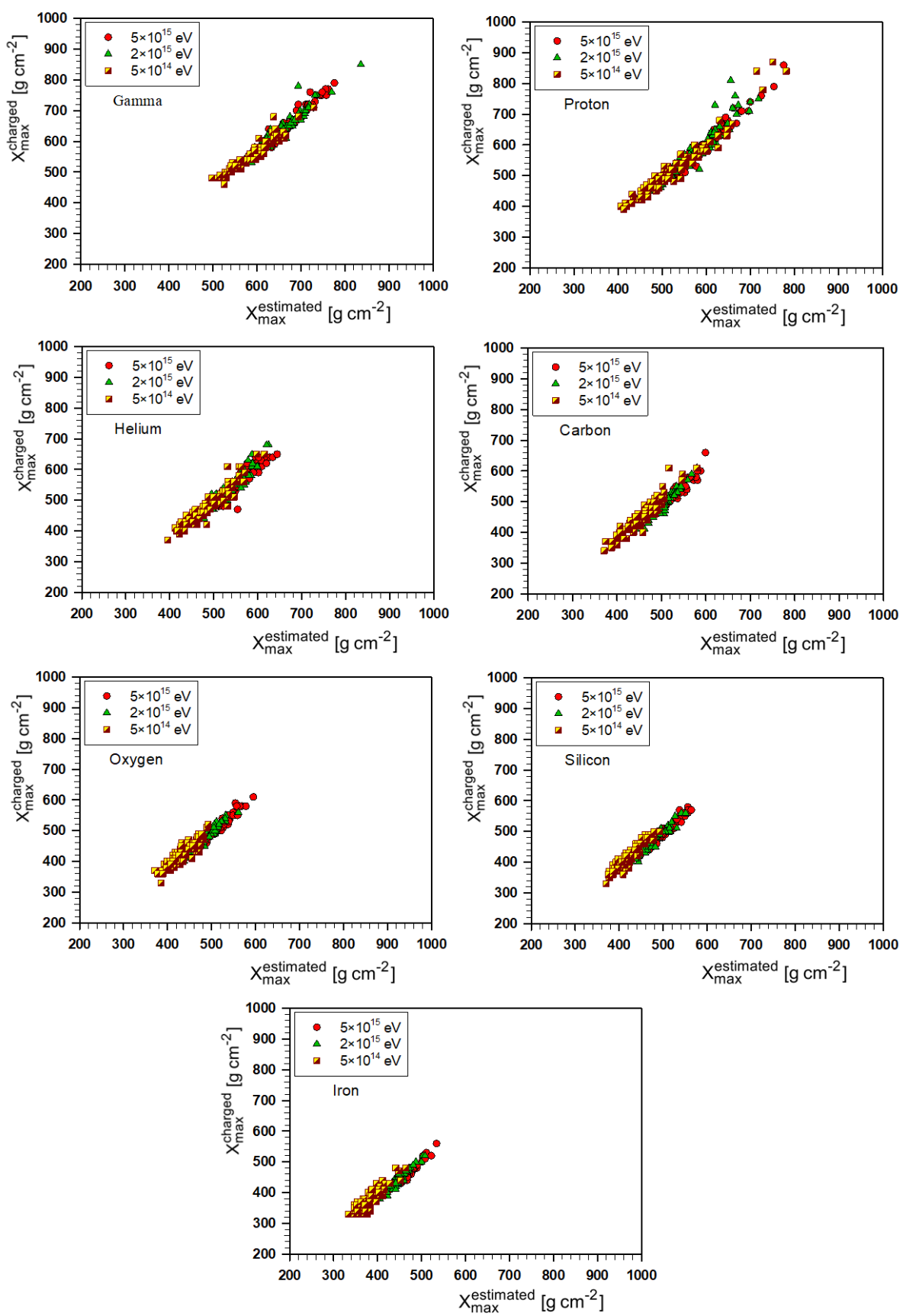

Figure 3: Comparison of shower maximum depths obtained from the longitudinal distribution of charged particles and those estimated by using equation 1 for simulated showers initiated by gamma rays, protons and the nuclei of helium, carbon, oxygen, silicon, and iron primary particles with $5 \times 10^{14} \mathrm{eV}, 2 \times 10^{15} \mathrm{eV}$, and $5 \times 10^{15} \mathrm{eV}$ energies. The number of simulated showers used in these figures is given in Table 2 . 

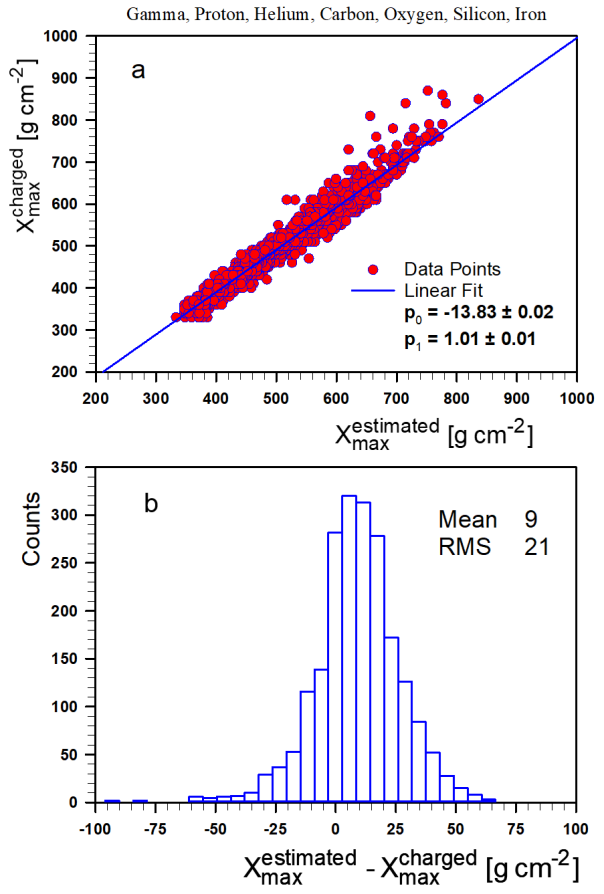

Figure 4: a) The actual maximum depths obtained from longitudinal distribution of charged particles have been compared with the maximum depths estimated by equation 1 for a set of 2100 simulated showers initiated by different primary particles, including gamma rays, proton, $\mathrm{He}, \mathrm{C}, \mathrm{O}, \mathrm{Si}$ and Fe with $5 \times 10^{14} \mathrm{eV}, 2 \times 10^{15} \mathrm{eV}, 5 \times 10^{15} \mathrm{eV}$ energies. The data points have been fitted to $X_{\text {max }}^{\text {charged }}=p_{0}+p_{1} X_{\text {max }}^{\text {estimated }} . b$ ) Histogram of the deviation of the estimated maximum depths from the actual ones, for the same data. 
Table 1: The number of simulated events used for fitting the data presented in Figure 2 to equation 1

\begin{tabular}{|c|c|c|c|c|c|c|c|}
\hline & \multicolumn{7}{|c|}{ No. of showers } \\
\hline Type $\backslash$ Energy & $10^{12} \mathrm{eV}$ & $10^{13} \mathrm{eV}$ & $5 \times 10^{13} \mathrm{eV}$ & $10^{14} \mathrm{eV}$ & $10^{15} \mathrm{eV}$ & $10^{16} \mathrm{eV}$ & $10^{17} \mathrm{eV}$ \\
\hline$\gamma$ & 100 & 100 & 100 & 100 & 100 & 100 & 20 \\
\hline$p$ & 100 & 100 & 100 & 100 & 100 & 100 & 20 \\
\hline $\mathrm{He}$ & 100 & 100 & 100 & 100 & 100 & 100 & 20 \\
\hline $\mathrm{C}$ & 100 & 100 & 100 & 100 & 100 & 100 & 20 \\
\hline $\mathrm{O}$ & 100 & 100 & 100 & 100 & 100 & 100 & 20 \\
\hline $\mathrm{Si}$ & 100 & 100 & 100 & 100 & 100 & 100 & 20 \\
\hline $\mathrm{Fe}$ & 100 & 100 & 100 & 100 & 100 & 100 & 20 \\
\hline
\end{tabular}

Table 2: Number of simulated events for testing equation 1 for $X_{\max }$ estimation of single showers in Figure 3

\begin{tabular}{|c|c|c|c|}
\hline & \multicolumn{3}{|c|}{ No. of showers } \\
\hline Type $\backslash$ Energy & $5 \times 10^{14} \mathrm{eV}$ & $2 \times 10^{15} \mathrm{eV}$ & $5 \times 10^{15} \mathrm{eV}$ \\
\hline$\gamma$ & 100 & 100 & 100 \\
\hline$p$ & 100 & 100 & 100 \\
\hline $\mathrm{He}$ & 100 & 100 & 100 \\
\hline $\mathrm{C}$ & 100 & 100 & 100 \\
\hline $\mathrm{O}$ & 100 & 100 & 100 \\
\hline $\mathrm{Si}$ & 100 & 100 & 100 \\
\hline $\mathrm{Fe}$ & 100 & 100 & 100 \\
\hline
\end{tabular}

\section{An investigation of immediate estimation of primary particles mass using $P$}

The main use of knowing the $X_{\max }$ of a shower is for the estimation of the mass of its primary particle. If one can find a model to relate the lateral distribution of Cherenkov radiation of an EASs to its primary mass, there will be no need to estimate $X_{\max }$. Therefore, we tried to find a model relating the shower primary particle mass to its steepness of the lateral Cherenkov radiation distribution, for a set of simulated extensive air showers. After the trial of a few models, we chose an exponential relation with two parameters, for its simplicity and suitable fit to the data:

$$
A=\exp (d \times P+g),
$$

where $A$ is the mass of the primary particle in atomic mass unit (a.m.u.), and $P$ is the steepness parameter. The model is only applicable to showers initiated by cosmic rays. The results of fitting the model to the simulated extensive air showers of different primary masses and energies have been shown in Figure 5. The fit parameters $d$ and $g$, which are presented in Table 5 are found to be energy dependent variables. It is worth noting that a linear dependence of $X_{\max }$ on $\ln A$ and $\ln E$ is a known fact in extensive air showers. For example, Kampert and Unger 15 have presented a linear equation, which reads as follows, after a parameter is renamed:

$$
\left\langle X_{\max }\right\rangle=f+D_{p} \ln (E / A),
$$



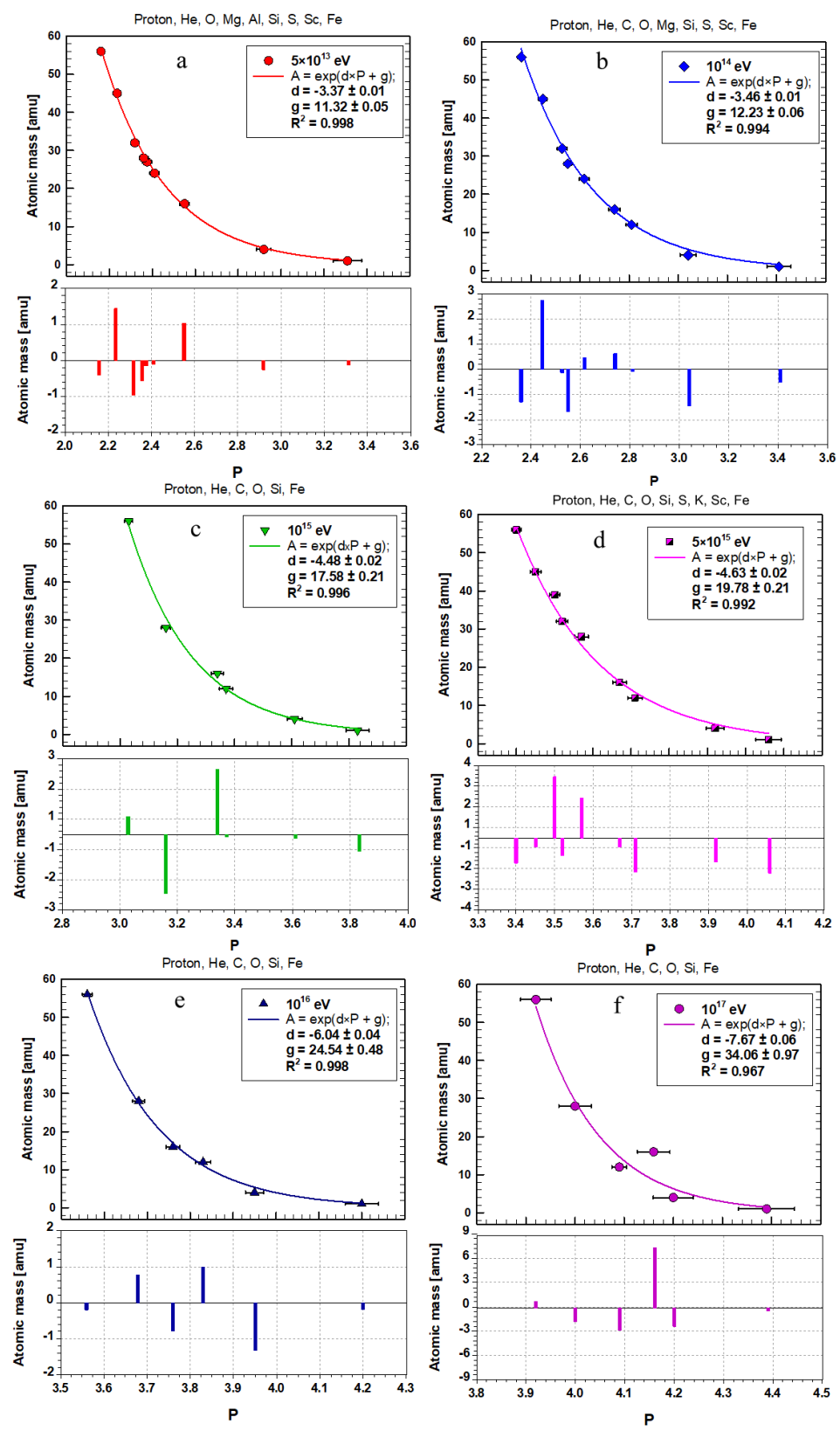

Figure 5: The results of fitting the equation 2 to a set of simulated extensive air showers initiated by different primary cosmic rays with $5 \times 10^{13} \mathrm{eV}, 10^{14} \mathrm{eV}, 10^{15} \mathrm{eV}, 5 \times 10^{15} \mathrm{eV}$, $10^{16} \mathrm{eV}$, and $10^{17} \mathrm{eV}$ energies. Each point is an average value among 100 showers, except for the $10^{17} \mathrm{eV}$ points which are average for 20 showers. The fit residuals are plotted below each figure. The number of simulated showers used in these plots is given in Table 4 
Table 3: The average values for the steepness of the lateral distribution of Cherenkov radiation, $P$, the maximum depths obtained from longitudinal distribution of charged particles, $X_{\text {max }}^{\text {charged }}$, the maximum depths estimated by using equation $1 X_{\text {max }}^{\text {estimated }}$, and the relative difference of the two maxima, $\delta=\frac{\left|X_{\max }^{\text {charged }}-X_{\max }^{\text {estimated }}\right|}{X_{\max }^{\text {charged }}} \mid$, for the set of simulated extensive air showers used in Figure 3

\begin{tabular}{|c|c|c|c|c|c|}
\hline Primary Particle & Primary Energy $[\mathrm{eV}]$ & $P=Q(100) / Q(200)$ & $X_{\max }^{\text {charged }}\left[\mathrm{g} / \mathrm{cm}^{2}\right]$ & $X_{\max }^{\text {estimated }}\left[\mathrm{g} / \mathrm{cm}^{2}\right]$ & $\delta$ \\
\hline \multirow[t]{3}{*}{$\gamma$-ray } & $5 \times 10^{14}$ & $4.13 \pm 0.03$ & $553 \pm 5.1$ & $587 \pm 4.7$ & 0.061 \\
\hline & $2 \times 10^{15}$ & $4.41 \pm 0.02$ & $613 \pm 5.9$ & $638 \pm 4.6$ & 0.041 \\
\hline & $5 \times 10^{15}$ & $4.55 \pm 0.02$ & $643 \pm 5.7$ & $663 \pm 4.3$ & 0.031 \\
\hline \multirow[t]{3}{*}{$p$} & $5 \times 10^{14}$ & $4.74 \pm 0.04$ & $519 \pm 9$ & $524 \pm 7.4$ & 0.010 \\
\hline & $2 \times 10^{15}$ & $3.96 \pm 0.04$ & $557 \pm 8.2$ & $558 \pm 6.1$ & 0.002 \\
\hline & $5 \times 10^{15}$ & $4.06 \pm 0.03$ & $574 \pm 7.5$ & $576 \pm 5.8$ & 0.003 \\
\hline \multirow[t]{3}{*}{$\mathrm{He}$} & $5 \times 10^{14}$ & $3.49 \pm 0.03$ & $477 \pm 5.7$ & $482 \pm 4.5$ & 0.010 \\
\hline & $2 \times 10^{15}$ & $3.72 \pm 0.02$ & $515 \pm 5.1$ & $519 \pm 3.9$ & 0.008 \\
\hline & $5 \times 10^{15}$ & $3.92 \pm 0.02$ & $549 \pm 5.2$ & $551 \pm 3.9$ & 0.004 \\
\hline \multirow[t]{3}{*}{$\mathrm{C}$} & $5 \times 10^{14}$ & $3.3 \pm 0.02$ & $448 \pm 5.2$ & $453 \pm 3.7$ & 0.011 \\
\hline & $2 \times 10^{15}$ & $3.52 \pm 0.02$ & $476 \pm 4$ & $486 \pm 3.1$ & 0.021 \\
\hline & $5 \times 10^{15}$ & $3.71 \pm 0.02$ & $509 \pm 4.1$ & $516 \pm 3.1$ & 0.014 \\
\hline \multirow[t]{3}{*}{$\mathrm{O}$} & $5 \times 10^{14}$ & $3.18 \pm 0.02$ & $427 \pm 3.8$ & $434 \pm 2.9$ & 0.016 \\
\hline & $2 \times 10^{15}$ & $3.47 \pm 0.02$ & $469 \pm 3.3$ & $478 \pm 2.6$ & 0.019 \\
\hline & $5 \times 10^{15}$ & $3.66 \pm 0.02$ & $503 \pm 3.7$ & $509 \pm 2.9$ & 0.012 \\
\hline \multirow[t]{3}{*}{$\mathrm{Si}$} & $5 \times 10^{14}$ & $3.08 \pm 0.02$ & $415 \pm 3.6$ & $419 \pm 2.5$ & 0.010 \\
\hline & $2 \times 10^{15}$ & $3.34 \pm 0.02$ & $450 \pm 3.7$ & $458 \pm 3.1$ & 0.018 \\
\hline & $5 \times 10^{15}$ & $3.57 \pm 0.02$ & $489 \pm 3.6$ & $494 \pm 2.8$ & 0.010 \\
\hline \multirow[t]{3}{*}{$\mathrm{Fe}$} & $5 \times 10^{14}$ & $2.83 \pm 0.02$ & $380 \pm 3.3$ & $383 \pm 2.3$ & 0.008 \\
\hline & $2 \times 10^{15}$ & $3.22 \pm 0.02$ & $431 \pm 3.1$ & $439 \pm 2.3$ & 0.019 \\
\hline & $5 \times 10^{15}$ & $3.4 \pm 0.01$ & $458 \pm 2.7$ & $466 \pm 2.1$ & 0.017 \\
\hline \multicolumn{5}{|r|}{$\langle\delta\rangle=$} & 0.016 \\
\hline
\end{tabular}

Table 4: Number of simulated showers used in Figure 5 for fitting equation 2

\begin{tabular}{|c|c|c|c|c|c|c|c|c|c|c|c|c|}
\hline Energies $[\mathrm{eV}]$ & $\mathrm{p}$ & $\mathrm{He}$ & $\mathrm{C}$ & $\mathrm{O}$ & $\mathrm{Si}$ & $\mathrm{Fe}$ & $\mathrm{Mg}$ & $\mathrm{Al}$ & $\mathrm{S}$ & $\mathrm{K}$ & $\mathrm{Sc}$ & $\mathrm{Sum}$ \\
\hline $5 \times 10^{13}$ & 100 & 100 & & 100 & 100 & 100 & 100 & 100 & 100 & & 100 & 900 \\
\hline $10^{14}$ & 100 & 100 & 100 & 100 & 100 & 100 & 100 & & 100 & & 100 & 900 \\
\hline $10^{15}$ & 100 & 100 & 100 & 100 & 100 & 100 & & & & & & 600 \\
\hline $5 \times 10^{15}$ & 100 & 100 & 100 & 100 & 100 & 100 & & & 100 & 100 & 100 & 900 \\
\hline $10^{16}$ & 100 & 100 & 100 & 100 & 100 & 100 & & & & & & 600 \\
\hline $10^{17}$ & 20 & 20 & 20 & 20 & 20 & 20 & & & & & & 120 \\
\hline \multicolumn{19}{|c|}{} \\
\hline
\end{tabular}

in which the elongation rate for proton-initiated showers $D_{p}=\frac{d\left\langle X_{\max }^{p}\right\rangle}{d \ln E}$ and $f$ are parameters that depend on the characteristics of hadronic interactions. By using equation 3 to replace $X_{\max }$ in equation 1 , we obtain a quadratic relation between $P$ and $\ln A$ :

$$
\ln A=-\frac{a}{D_{p}} P^{2}-\frac{b}{D_{p}} P+\frac{D_{p} \ln E+f-c}{D_{p}},
$$

in which $a, b$, and $c$ are parameters of equation 1. The last term in equation 4 is clearly energy dependent . On the other hand, even though $D_{p}$ has almost a constant value of $25 \mathrm{~g} / \mathrm{cm}^{2}$ (see ref. [15]), the coefficient of the first term in the linear model will bacome energy dependent if we ignore the first term in equation 4 to obtain the simpler linear model given in equation 2. Thus, equation 2 can be represented in another form:

$$
A=\exp \left(\alpha\left(E_{0}\right) \times P+\beta\left(E_{0}\right)\right)
$$


Table 5: The fit parameters and coefficient of determination for fitting the equation $A=$ $\exp (d \times P+g)$ with the data presented in the Figure $5 E_{0}$ is the energy of showers.

\begin{tabular}{|c|c|c|c|c|c|c|}
\hline \hline$E_{0}[\mathrm{eV}]$ & $5 \times 10^{13}$ & $10^{14}$ & $10^{15}$ & $5 \times 10^{15}$ & $10^{16}$ & $10^{17}$ \\
\hline$d$ & $-3.37 \pm 0.01$ & $-3.46 \pm 0.01$ & $-4.48 \pm 0.02$ & $-4.63 \pm 0.02$ & $-6.04 \pm 0.04$ & $-7.67 \pm 0.06$ \\
$g$ & $11.32 \pm 0.05$ & $12.23 \pm 0.06$ & $17.58 \pm 0.21$ & $19.78 \pm 0.21$ & $25.54 \pm 0.48$ & $34.06 \pm 0.98$ \\
$R^{2}$ & 0.998 & 0.994 & 0.996 & 0.992 & 0.998 & 0.967 \\
\hline
\end{tabular}

in which $E_{0}$ is the energy of the shower. A quadratic function of $\ln E_{0}$ is found to be suitable for $\alpha\left(E_{0}\right)$ and $\beta\left(E_{0}\right)$ :

$$
\begin{aligned}
& \alpha\left(E_{0}\right)=a_{1} \ln E_{0}^{2}+b_{1} \ln E_{0}+c_{1} \\
& \beta\left(E_{0}\right)=a_{2} \ln E_{0}^{2}+b_{2} \ln E_{0}+c_{2}
\end{aligned}
$$

in which $E_{0}$ is in $\mathrm{TeV}$. The fit parameters for equations 6 and 7 are given in Table 6. Thus, equation 5 is applicable for primary mass estimation if the shower energy is known. Fortunately, previous research has shown that the shower energy can be estimated from the surface density of Cherenkov radiation at a fixed distance from the shower core. For example, Lubsandorzhiev [8] has presented the following relation between the shower energy and the Cherenkov photon density at $175 \mathrm{~m}$ from the shower core:

$$
E_{0}=400 \times\left(Q_{175}\right)^{0.95},
$$

where $Q_{175}$ is photon density in $\mathrm{cm}^{-2} \mathrm{eV}^{-1}$, and $E_{0}$ is the shower energy in $\mathrm{TeV}$. This has been utilized by Tunka Collaboration for the shower energy estimation. Therefore, we used equation 8 to estimate the energies and then equation 5 to estimate the primary masses of another set of 1900 simulated extensive air showers. The number of simulated showers for each type-energy is given in Table 7. It should be emphasized that the new set of showers is different from that of showers used for the estimation of fit parameters presented in Table 5 and Table 6. The histogram of deviation of the estimated primary masses from the

Table 6: The fit parameters for equations 6 and 7, evaluated from the data presented in Table 5

\begin{tabular}{|c|c|c|c|c|}
\hline \hline Equation $[6]$ & $a_{1}$ & $b_{1}$ & $c_{1}$ & $R^{2}$ \\
\hline Equation [ & $-0.062 \pm 0.005$ & $0.384 \pm 1.076$ & $-3.941 \pm 12.97$ & 0.964 \\
\hline & $a_{2}$ & $b_{2}$ & $c_{2}$ & $R^{2}$ \\
\hline & $0.261 \pm 0.005$ & $-1.035 \pm 1.076$ & $11.453 \pm 12.97$ & 0.984 \\
\hline
\end{tabular}

actual masses is depicted in Figure 6. The mean of the deviation distribution is close to zero. This means the method can discriminate the average primary mass of cosmic rays. However, the large value of the RMS implies a statistical error of 19 a.m.u. for a single event mass estimation. 
Table 7: The number of simulated showers used in Figure 6 for the test of atomic mass estimation by equation 5

\begin{tabular}{|c|c|c|c|c|c|c|c|c|c|c|c|c|}
\hline Energies $[\mathrm{eV}]$ & $\mathrm{p}$ & $\mathrm{He}$ & $\mathrm{C}$ & $\mathrm{O}$ & $\mathrm{Si}$ & $\mathrm{Fe}$ & $\mathrm{Mg}$ & $\mathrm{Al}$ & $\mathrm{S}$ & $\mathrm{K}$ & $\mathrm{Sc}$ & Sum \\
\hline $10^{14}$ & 50 & 50 & 50 & 50 & 50 & 50 & & & & & & 300 \\
\hline $5 \times 10^{14}$ & & & 100 & 100 & & 100 & 100 & 100 & 100 & 100 & 100 & 800 \\
\hline $10^{15}$ & 50 & 50 & 50 & 50 & 50 & 50 & & & & & & 300 \\
\hline $2 \times 10^{15}$ & & & 100 & 100 & & 100 & & & & 100 & 100 & 500 \\
\hline \multicolumn{10}{|c|}{ Total sum } & 1900 \\
\hline
\end{tabular}

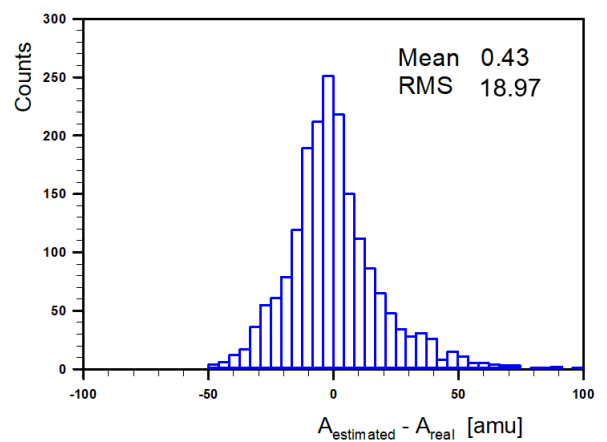

Figure 6: The distribution of differences between the actual masses of the primary particles and the estimated masses obtained by application of equation 5 The results are based on event-by-event evaluation for 1900 simulated extensive air showers initiated by primary masses from $A=1$ to $A=56$ and with energies from $10^{14} \mathrm{eV}$ to $2 \times 10^{15} \mathrm{eV}$. The number of simulated showers for each type-energy is given in Table 7

\section{Impact of the hadronic interaction models on the results}

All the analyses presented in previous sections were based on showers simulated using QGSJETII and GHEISHA hadronic interaction models. In order to test the impact of hadronic interaction models on the proposed method of $X_{\max }$ estimation, we simulated a set of $10^{14}, 10^{15}$ and $10^{16} \mathrm{eV}$ showers initiated by five different primary masses with EPOS-LHC model for high energy, and UrQMD model for low energy hadronic interactions. The numbers of simulated showers are given in Table 8. The steepness parameter of each shower was then used in

Table 8: Number of simulated showers with EPOS-LHC+UrQMD hadronic models.

\begin{tabular}{|c|c|c|c|}
\hline & \multicolumn{3}{|c|}{ No. of showers } \\
\hline Type $\backslash$ Energy & $10^{14} \mathrm{eV}$ & $10^{15} \mathrm{eV}$ & $10^{16} \mathrm{eV}$ \\
\hline$\gamma$ & 100 & 100 & 30 \\
\hline$p$ & 100 & 100 & 30 \\
\hline $\mathrm{He}$ & 100 & 100 & 30 \\
\hline $\mathrm{C}$ & 100 & 100 & 30 \\
\hline $\mathrm{Fe}$ & 100 & 100 & 30 \\
\hline
\end{tabular}

equation 1 to estimate its $X_{\max }$. It should be noted that the parameters of equation 1 are obtained by using showers generated through QGSJETII+GHISHA 
models. A comparison of estimated depths with those obtained from longitudinal distribution of charged particles of the showers, together with the similar results for showers of the same type-energy, generated by QGSJETII+GHEISHA models, is presented in Figure 7. The average values of estimated and actual $X_{\max }$ for the EPOS-UrQMD showers are given in Table 9 A histogram of $X_{\text {max }}^{\text {estimated }}-X_{\text {max }}^{\text {charged }}$ is shown in Figure 8. Despite differences in the slopes and offsets of the linear fits in the two sets of hadronic models presented in Figure 7 the statistical error of the estimated $X_{\max }$ of EPOS-UrQMD showers, which is inferred from Figure 8 to be about $30 \mathrm{~g} / \mathrm{cm}^{2}$, is lower than the difference between the average $X_{\max } \mathrm{s}$ of low mass and high mass cosmic rays. The application of equation 2 has also been tested on the EPOS+UrQMD showers initiated by proton, helium, carbon and iron. The histogram of deviation of estimated mass from actual mass is shown in Figure 9. The RMS in the estimated mass deviations is comparable to the one obtained for QGSJETII+GHEISHA showers (see Figure 6). Although different hadronic models can generate slightly different $X_{\max }$ for showers of the same type-energy, it seems that the relation between the $X_{\max }$ and the lateral distribution of Cherenkov radiation is not seriously affected. In fact, hadronic interaction models are expected to diverge at energies higher than $10^{17} \mathrm{eV}$ where no accelerator data are available. As we did not have enough computation time to simulate higher energy showers, the presented test is limited to $\left[10^{14}, 10^{16}\right] \mathrm{eV}$ energy range. Further tests should be done for higher energies. The results of the maximum depths for showers simulated through EPOS-LHC and QGSJETII hadronic models in energies above $10^{17} \mathrm{eV}$ reported by Yushkov et al. show that the difference between average $X_{\max }$ in the two models is lower than $30 \mathrm{~g} / \mathrm{cm}^{2}$ [16].

Table 9: The average values for the steepness of the lateral distribution of Cherenkov radiation, $P$, the maximum depths obtained from longitudinal distribution of charged particles, $X_{\text {max }}^{\text {charged }}$, the maximum depths estimated by using equation $1 . X_{\text {max }}^{\text {estimated }}$, and the relative difference of the two maxima, $\delta=\frac{\left|X_{\max }^{\text {charged }}-X_{\max }^{\text {estimated }}\right|}{X_{\max }^{\text {charged }}} \mid$, for the showers simulated with EPOS+UrQMD hadronic interaction models. The number of simulated showers used for calculation of these data is given in Table 8

\begin{tabular}{|c|c|c|c|c|c|}
\hline Primary Particle & Primary Energy $[\mathrm{eV}]$ & $P=Q(100) / Q(200)$ & $X_{\text {max }}^{\text {charg }}\left[\mathrm{g} / \mathrm{cm}^{2}\right]$ & $X_{\text {max }}^{\text {estimated }}\left[\mathrm{g} / \mathrm{cm}^{2}\right]$ & $\delta$ \\
\hline$\gamma$-ray & $10^{14}$ & $3.87 \pm 0.04$ & $510 \pm 6.6$ & $544 \pm 6.8$ & 0.067 \\
& $10^{15}$ & $4.28 \pm 0.03$ & $579 \pm 5.8$ & $614 \pm 5.9$ & 0.061 \\
& $10^{16}$ & $4.63 \pm 0.04$ & $660 \pm 9.5$ & $678 \pm 6.9$ & 0.027 \\
\hline$p$ & $10^{14}$ & $3.41 \pm 0.05$ & $484 \pm 10$ & $472 \pm 7.6$ & 0.025 \\
& $10^{15}$ & $3.87 \pm 0.05$ & $543 \pm 9.8$ & $546 \pm 8.1$ & 0.004 \\
& $10^{16}$ & $4.14 \pm 0.04$ & $590 \pm 10.6$ & $590 \pm 7.5$ & 0. \\
\hline $\mathrm{He}$ & $10^{14}$ & $3.14 \pm 0.03$ & $437 \pm 6.1$ & $430 \pm 4.9$ & 0.016 \\
& $10^{15}$ & $3.48 \pm 0.02$ & $531 \pm 5.8$ & $528 \pm 4.9$ & 0.006 \\
& $10^{16}$ & $2.86 \pm 0.02$ & $398 \pm 5.5$ & $389 \pm 3.4$ & 0.009 \\
\hline $\mathrm{C}$ & $10^{14}$ & $3.48 \pm 0.02$ & $479 \pm 4.5$ & $479 \pm 3.3$ & 0. \\
& $10^{15}$ & $3.87 \pm 0.04$ & $546 \pm 8.2$ & $543 \pm 6.4$ & 0.005 \\
\hline $\mathrm{Fe}$ & $10^{16}$ & $2.35 \pm 0.01$ & $338 \pm 2.8$ & $321 \pm 1.7$ & 0.050 \\
& $10^{14}$ & $3.08 \pm 0.01$ & $423 \pm 2.6$ & $420 \pm 1.9$ & 0.007 \\
& $10^{15}$ & $3.62 \pm 0.02$ & $501 \pm 4.6$ & $501 \pm 3.3$ & 0. \\
\hline
\end{tabular}




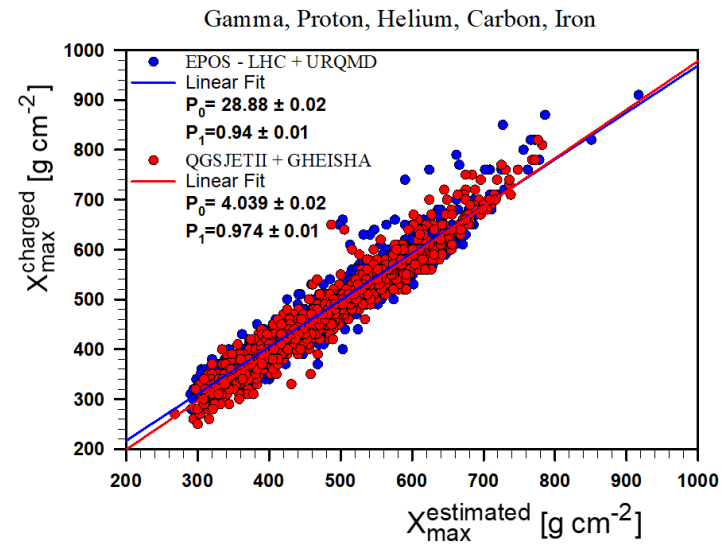

Figure 7: A comparison of $X_{\max }^{\text {estimated }}$ obtained by using equation 1 with $X_{\text {max }}^{\text {chared }}$ for showers initiated by gamma ray, proton, helium, carbon and iron primaries of $10^{14}, 10^{15}$ and $10^{16} \mathrm{eV}$ energies. The blue dots are for showers generated with EPOS-LHC+UrQMD models, and the red dots are for those generated by QGSJETII+GHEISHA models. Each population has been fit to a linear model $X_{\text {max }}^{\text {charged }}=p_{0}+p_{1} X_{\text {max }}^{\text {estimated }}$. The corresponding fit parameters are given in the legend. The number of showers used for each type-energy-model can be found in Tables 1 and 8

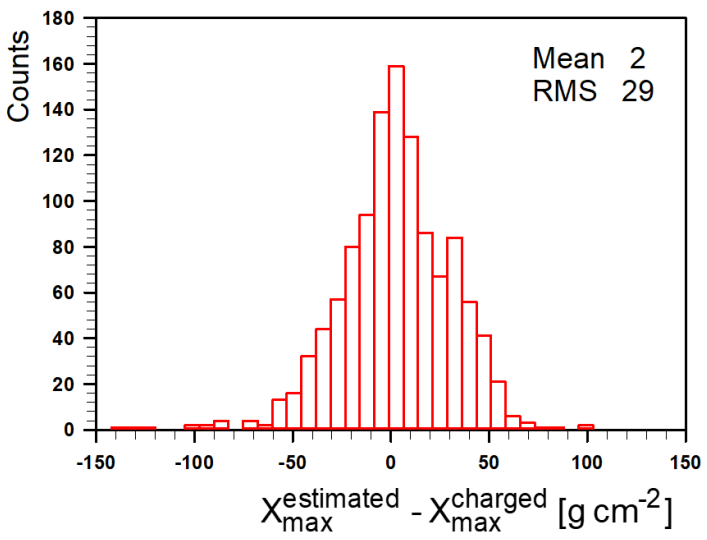

Figure 8: A histogram of difference of $X_{\max }^{\text {estimated }}$ with $X_{\max }^{\text {charged }}$ for showers initiated by gamma ray, proton, helium, carbon and iron primaries of $10^{14}, 10^{15}$ and $10^{16} \mathrm{eV}$ energies generated with EPOS-LHC+UrQMD hadronic models. The number of showers used for each type-energy can be found in Table 8 


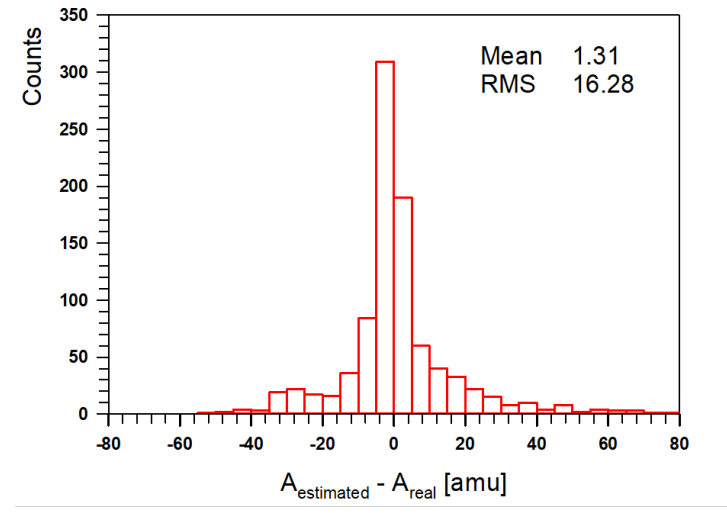

Figure 9: The distribution of differences between the actual masses of the primary particles and the estimated masses obtained by application of equation 2 The results are based on event-by-event evaluation for 920 simulated extensive air showers initiated by proton, helium, carbon and iron primaries of $10^{14}, 10^{15}$ and $10^{16} \mathrm{eV}$ energies, which were generated with EPOS-LHC+UrQMD hadronic models. The number of showers used for each type-energy can be found in Table 8

\section{Conclusion}

One of the main goals of observing extensive air showers is the estimation of the mass of their primary cosmic rays. The mass is usually estimated by measuring the shower maximum depth with air fluorescence detectors [7, or by measuring the muon to electron population ratio with an array of particle detectors [17. Since the Cherenkov wave front of an extensive air shower is mainly generated by high energy electrons, the depth of maximum of a shower is the origin of the most of the radiation. The lateral distribution of the Cherenkov radiation on the ground is, then, affected by variation of the shower maximum depth. Although the details of Cherenkov lateral distribution depend on the variation of the energy distribution of the secondary electrons, and structure of the atmosphere, the Monte Carlo simulation technique provides the appropriate means to investigate the phenomena. Researchers in Tunka experiment have used the slope parameter of the lateral distribution of Cherenkov radiation in extensive air showers, for the estimation of the height of the shower maximum. The estimated height, then, has to be converted to atmospheric depth in order to be used for the primary mass estimation. Here, we indicated that the maximum depth could be immediately related to the slope parameter, with no need to the intermediate step of maximum height estimation. The maximum depths estimated with the new method are more accurate. This, in turn, can improve the accuracy of the cosmic ray mass composition estimated by Cherenkov wave front experiments, like Tunka-133 array [18. We have also tested a new relation between the primary mass and the slope parameter. The method has been tested for the estimation of the primary masses from the measured slope of the lateral Cherenkov radiation of simulated extensive air showers. Although the idea is 
useful for average mass estimation, the statistical errors are large. This prevents the application of the method for an accurate estimation of the primary mass of a single shower. It should be mentioned that the detector array response has not been taken into account in our analysis. In reality, the estimated position of a shower core and the photon densities in an array have large instrumental errors. These errors can reduce the accuracy of the results. It is worth noting that due to limited computation time, our simulated data were restricted to the vertical showers. Both shower zenith angle and detector responses can affect the accuracy of the results. Further investigations should take account of a specific detector array response and dependence of Cherenkov lateral distribution on the shower zenith angle.

\section{Acknowledgements}

Most of the simulation in this work has been performed with the HPC supercomputer at Imam Khomeini International University (IKIU). We are grateful to the manager and the staff of HPC facility of IKIU for their support.

\section{References}

[1] J. Bellido, Pierre Auger Collaboration, in: 35th International Cosmic Ray Conference (ICRC2017), volume 301 of International Cosmic Ray Conference, p. 506.

[2] S. F. Berezhnev, et al., Nucl. Instrum. Meth. A 692 (2012) 98-105. doi:10. 1016/j.nima.2011.12.091, arXiv:1201.2122.

[3] A. Corstanje, S. Buitink, H. Falcke, B. M. Hare, J. R. Hörandel, T. Huege, G. K. Krampah, P. Mitra, K. Mulrey, A. Nelles, H. Pandya, J. P. Rachen, O. Scholten, S. ter Veen, S. Thoudam, G. Trinh, T. Winchen, arXiv e-prints (2021) arXiv:2103.12549. arXiv:2103.12549

[4] D. Chernov, et al., Int. J. Mod. Phys. A 20 (2006) 6799-6801. doi 10.1142/ S0217751X05030120, arXiv:astro-ph/0411139.

[5] R. Takeishi, Telescope Array Collaboration, in: European Physical Journal Web of Conferences, volume 182 of European Physical Journal Web of Conferences, p. 02122. doi:10.1051/epjconf/201818202122.

[6] L. B. Arbeletche, V. de Souza, Astroparticle Physics 116 (2020) 102389. doi:10.1016/j .astropartphys .2019.102389, arXiv:1903.03174.

[7] R. U. Abbasi, et al, The Astrophysical Journal 76 (2018) 858-885. doi: 10 . 3847/1538-4357/aabad7.

[8] B. K. Lubsandorzhiev (TUNKA), Nucl. Instrum. Meth. A 595 (2008) 7376. doi:10.1016/j.nima.2008.07.054. 
[9] T. Stanev, High energy cosmic rays, Springer Science \& Business Media, Heidelberg, 2010.

[10] D. Heck, J. Knapp, J. N. Capdevielle, G. Schatz, T. Thouw, CORSIKA: A Monte Carlo code to simulate extensive air showers, FZKA-6019, 1998.

[11] S. Ostapchenko, Nucl. Phys. B Proc. Suppl. 151 (2006) 143-146. doi 10. 1016/j.nuclphysbps.2005.07.026. arXiv:hep-ph/0412332.

[12] H. Fesefeldt, The Simulation of Hadronic Showers: Physics and Applications, PITHA-85-02, 1985.

[13] Pierog, T. and Karpenko, Iu. and Katzy, J. M. and Yatsenko, E. and Werner, K., Phys. Rev. C 92 (2015) 034906. URL: https://link.aps.org/doi/10.1103/PhysRevC.92.034906 doi $\{10.1103 /$ PhysRevC.92.034906\}.

[14] S. A. Bass, et al, Progress in Particle and Nuclear Physics 41 (1998) 255369. URL: https://www.sciencedirect.com/science/article/pii/ S0146641098000581. doi:\{https://doi.org/10.1016/S0146-6410(98) 00058-1\}.

[15] K.-H. Kampert, M. Unger, Astroparticle Physics 35 (2012) 660678. URL: https://www.sciencedirect.com/science/article/pii/ S0927650512000382 doi/https://doi.org/10.1016/j.astropartphys. 2012.02 .004

[16] A. Yushkov, J. Bellido, J. Belz, V. de Souza, W. Hanlon, D. Ikeda, P. Sokolsky, Y. Tsunesada, M. Unger, Pierre Auger Collaboration, Telescope Array Collaboration, in: European Physical Journal Web of Conferences, volume 210 of European Physical Journal Web of Conferences, p. 01009. doi:10.1051/epjconf/201921001009, arXiv:1905.06245.

[17] D. Purmohammad, Iranian Journal of Physics Research 13 (2013) 310-310. URL: http://ijpr.iut.ac.ir/article_1045.html.

[18] V. Prosin, et al., Nucl. Instrum. Meth. A 756 (2014) 94-101. doi 10.1016/ j.nima.2013.09.018 